\title{
Lick Sodium Laser Guide Star: Performance During the 1998 LGS Observing Campaign
}

\author{
Don Gavel \\ Herb Friedman \\ Brian Bauman
}

This paper was prepared for submittal to the 44th Annual Mecting of the International Symposium on

Optical Science, Engineering, and Instrumentation

Denver, $\mathrm{CO}$

July 18-23, 1999

July 19, 1999

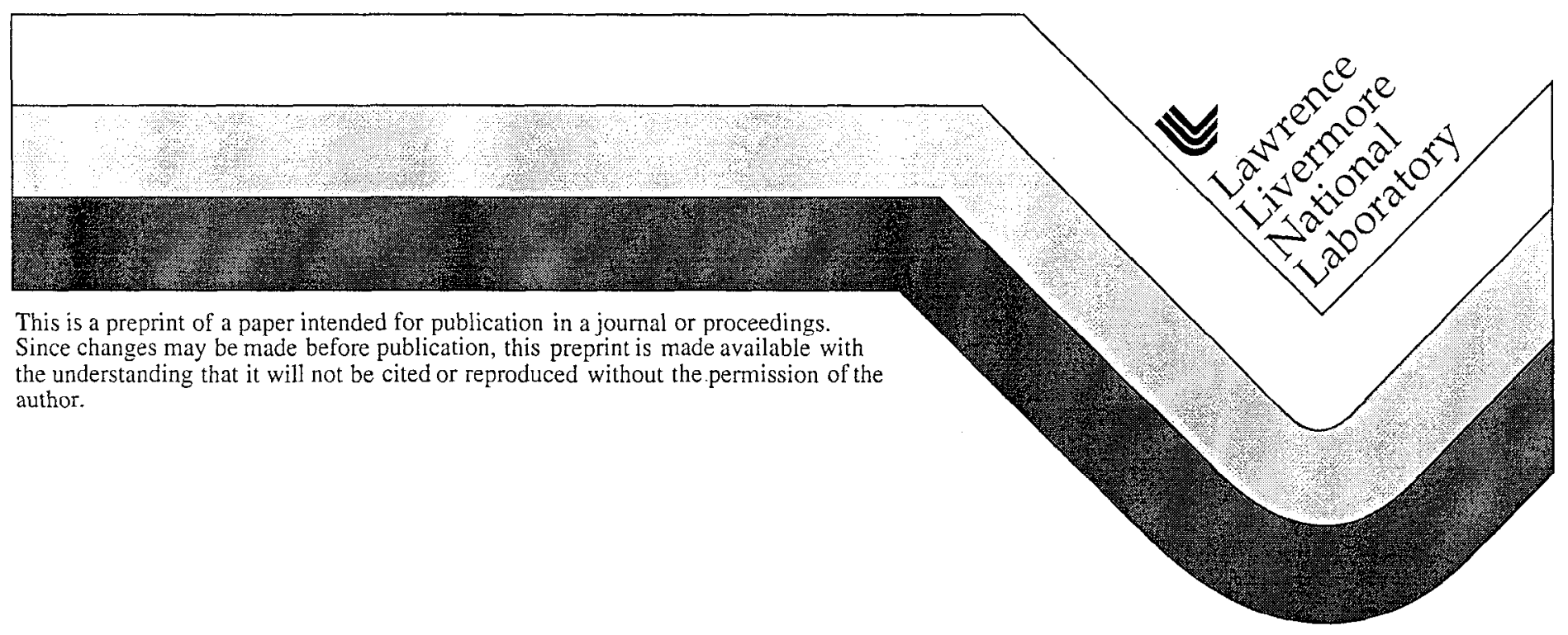




\section{DISCLAIMER}

This document was prepared as an account of work sponsored by an agency of the United States Government. Neither the United States Government nor the University of California nor any of their employees, makes any warranty, express or implied, or assumes any legal liability or responsibility for the accuracy, completeness, or usefulness of any information, apparatus, product, or process

disclosed, or represents that its use would not infringe privately owned rights. Reference herein to any specific commercial product, process, or service by trade name, trademark, manufacturer, or otherwise, does not necessarily constitute or imply its endorsement, recommendation, or favoring by the United States Government or the University of California. The views and opinions of authors expressed herein do not necessarily state or reflect those of the United States Government or the University of California, and shall not be used for advertising or product endorsement purposes. 


\title{
Lick Sodium Laser Guide Star: Performance During the 1998 LGS Observing Campaign
}

\author{
Don Gavel, Herb Friedman, and Brian Bauman \\ Lawrence Livermore National Laboratory \\ 7000 East Avenue, MS L-395, Livermore, CA 94550
}

\begin{abstract}
The performance of a sodium laser guide star adaptive optics system depends crucially on the characteristics of the laser guide star in the sodium layer. System performance is quite sensitive to sodium layer spot radiance, that is, return per unit sterradian on the sky, hence we have been working to improve projected beam quality via improvements to the laser and changes to the launched beam format. The laser amplifier was reconfigured to a "bounce-beam" geometry, which considerably improves wavefront quality and allows a larger round instead of square launch beam aperture. The smaller beacon makes it easier to block the unwanted Rayleigh light and improves the accuracy of Hartmann sensor wavefront measurements in the AO system. We present measurements of the beam quality and of the resulting sodium beacon and compare to similar measurements from last year.
\end{abstract}

Keywords: $\quad$ Sodium Laser Guide Star, Laser Guide Star

\section{INTRODUCTION}

The LLNL Lick LGS AO system has shown that sodium beacon AO for astronomy is practical. In 1996 and 1997, the LGS AO strehl was consistently in the 0.1 range - showing diffraction-limited cores on corrected images and significant improvement over open-loop and tip tilt only images. In late 1998, the LGS AO system demonstrated a Strehl of 0.42 during reasonably good seeing (1/2 arcsecond) at Lick ${ }^{1}$. Our goal is now to make LGS AO as routine as NGS AO for astronomers, which requires that the laser perform reliably and that a reasonable guidestar is formed most seasons of the year, even during periods when the mesospheric sodium abundance is low. To make this happen, every precious Watt from the laser must be put to good use, and this is done by projecting the laser into as tight an LGS spot as possiblc as the analysis in section 2 will show.

Performance measurements of the laser and of the laser guide star spot in 1998 showed significant improvement over the situation in $1997^{2}$. The improvement is attributed mostly to the introduction of the "bounce-beam" geometry in the final laser amplifier (section 3) which considerably improved laser wavefront quality. The LGS spot size is not as small as it should be however. Assuming a perfect wavefront, and given the size of our projection aperture and the measured seeing conditions, the spot size could still be reduced by a factor of 2 to 3 . We can attribute the non-optirnum spot size to still somewhat imperfect laser wavefront and, more significantly, to difficulties in aligning the launch telescope. The later is discussed in section 6. 


\section{DEPENDENCE OF AO PERFORMANCE ON THE SIZE OF THE GUIDESTAR}

The accuracy of the Hartmann wavefront sensor depends on the signal-to-noise ratio in each subaperture and on the focal plane size of the guide star spot. For a quad-cell centroiding algorithm, the centroid error is given by

$$
\sigma_{\text {noise }}=\eta \chi \frac{2 \pi d}{\lambda} \frac{\iint_{-\infty-\infty}^{\infty} \int(x, y) d x d y}{\sqrt{2} \int_{-\infty}^{\infty} f(0, y) d y} \times \frac{1}{\mathrm{SNR}}
$$

where $\sigma_{\text {noise }}$ is rms wavefront error, in radians of phase, due to imperfect measurement of Hartmann centroids, $f(x, y)$ is the distribution of guide star intensity at the focal plane of the wavefront sensor, $x$ and $y$ are angle coordinates on the sky, $d$ is the Hartmann subaperture size, and $\eta$ and $\chi$ are unitless constants of order unity that depend on the exact nature of the wavefront reconstruction matrix and control loop parameters respectively. The ratio of integrals defines a characteristic spot size, which we refer to as the spot size factor. Clearly a smaller spot size factor is desirable; a factor of 2 improvement in spot size of the laser guide star for example, is equivalent to a factor of 4 increase in laser power!

The spot size factor can be measured in both the natural and laser guide star cases from high resolution images taken with a science grade CCD camera located at a focus of the AO system. Natural guide stars (point sources) in good seeing have a spot size factor of about 0.5 arcseconds. The laser guide star has a measured spot size of about 1.7 arcseconds.

\section{IMPROVEMENTS TO THE LICK LASER IN 1998}

The Laser at Lick observatory ${ }^{3}$ consists of a 20 Watt dye amplifier mounted on the telcscope truss. This is fed by a seed beam from a dye master oscillator, which is tuned to the Sodium D2 line, and by 200 Watts of pump laser light laser coming from a set of doubled NdYg lasers housed in the observatory basement.

Originally, the Sodium beam passed straight through the final amplifier dye cell, with pump light illuminating from two sides of the cell. A major innovation developed for the Keck laser $^{4}$ was incorporated into the Lick system this year: to bounce the beam once off an inside surface of the final amplifier dye cell and to pump from only one side. The symmetric inversion of the beam halfway through the pump profile greatly reduces pumped-induced aberrations.

An important additional benefit for the Lick system is the change from a square format to a circular launch beam. Originally, becausc of the ccll gcometry, the straight through beam was required to be square so that it would fill the dye cell to prevent broad band amplified spontaneous emission from unseeded but pumped dye. The bounce-beam geometry allows a circular beam to reasonably fill the dye cell. The result is a $25 \mathrm{~cm}$ diameter circular launch aperture instead of a $20 \times 20 \mathrm{~cm}$ square one, which reduces the far-field diffraction of the launched beam. 


\section{LASER WAVEFRONT DIAGNOSTIC SYSTEM}

The Lick laser has a supplementary optical bench, located further up the telescope truss from the final amplifier, containing three beam diagnostic sensors to aid in the alignment through the dye cells and to provide a means of measuring laser wavefront quality. Figure 1 shows a diagram of the diagnostic optical paths. Two video cameras view the near-field and far-field beam patterns, respectively. A third camera, with collimating optics and a lenslet array, forms a Hartmann sensor. Images from the near and far-field cameras are shown in Figures 2 and 3.

Far field imaging with the 8-bit video camera requires two exposures to extend this data to a useful dynamic range. One image captures the central lobe without saturation and another saturates the central lobe but captures the low intensity wings of the diffraction pattern. The two-exposures are later combined with appropriate weighting factors. A disadvantage of this method is that it is sensitive to beam intensity fluctuation between exposures. Later this year we plan to upgrade to a 12-bit camera, in which case we will be able to capture full dynamic range data in a single exposure.

The Hartmann sensor, while providing a qualitative map of the beam wavefront very useful in the dye cell alignment stages, has not been satisfactorily calibrated for quantitative wavefront measurement. Precision calibration of this sensor is slated for later this year.

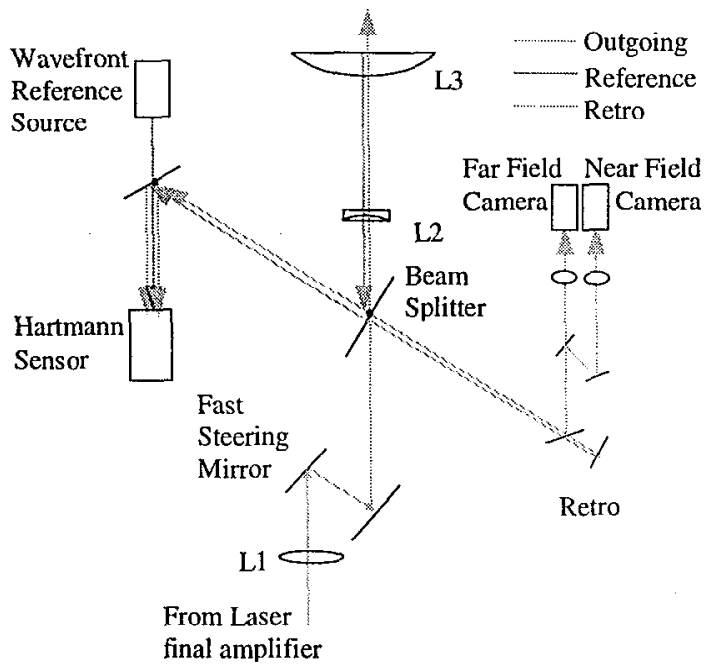

Figure 1. Lick guide star laser diagnostic system

\section{LASER AND AO SYSTEM PERFORMANCE DURING THE 1998 AO RUNS}

Images of near and far field beam profile show very high beam quality at low power, with some degradation as the laser is boosted to 18 Watts output power. The near field camera image plane is conjugate to the final optic, L3, so it provides a map of launch beam intensity (Figure 2). The diameter of the beam is about $25 \mathrm{~cm}$ on the $30 \mathrm{~cm}$ diameter output lens. 
The far field image (Figure 3) is an indication of the beam's diffraction pattern, and would be the illumination profile at the sodium layer were there no atmospheric turbulence. Under typical seeing conditions, this far field pattern would be convolved twice by the point spread function of the atmosphere, once for the upgoing path, once for the downgoing path (not accounting for possible saturation effects of the sodium layer response). In 1 arcsecond seeing therefore we would expect to see the 0.5 arcsecond laser blurred out to about 1.5 arcseconds.

Our images with through the receiving (Lick 3 meter Shane) telescope show a much larger guide star spot however (Figure 4). The spot is approximately 2.5 arcseconds full-width-half-maximum, and has a spot size factor (from equation 1) of 1.7 arcseconds. By adjusting the launch telescope (L2-L3 pair) through focus, a characteristic astigmatism pattern becomes apparent. We attribute this pattern, and the consequent large guide star spot, to mis-centering of the beam through the final launch telescope. The design tolerances on this telescope are such that significant aberration is introduced if the beam is a few millimeters off the center of L2. 'I his amount of decentering had apparently been introduced earlier by steering the laser (using M1 and L2, see Figure 1) to align it with the Hartmann sensor in the adaptive optics system, without realizing that the telescope aberrations would be so large. The remedy is to re align the telescope, then redo the pointing adjustments in such a way to preserve proper centering on L2 and L3.

Nevertheless, the improvements to the laser itself improved the guide star measurably, as the graph of encircled energy, Figure 5, shows. The 50\% energy radius improved from 1.5 to 1.0 arcseconds and the $80 \%$ energy radius from 3.4 to 2.2 arcseconds. The spot size factor improved from 2.97 to 1.7 arcseconds.
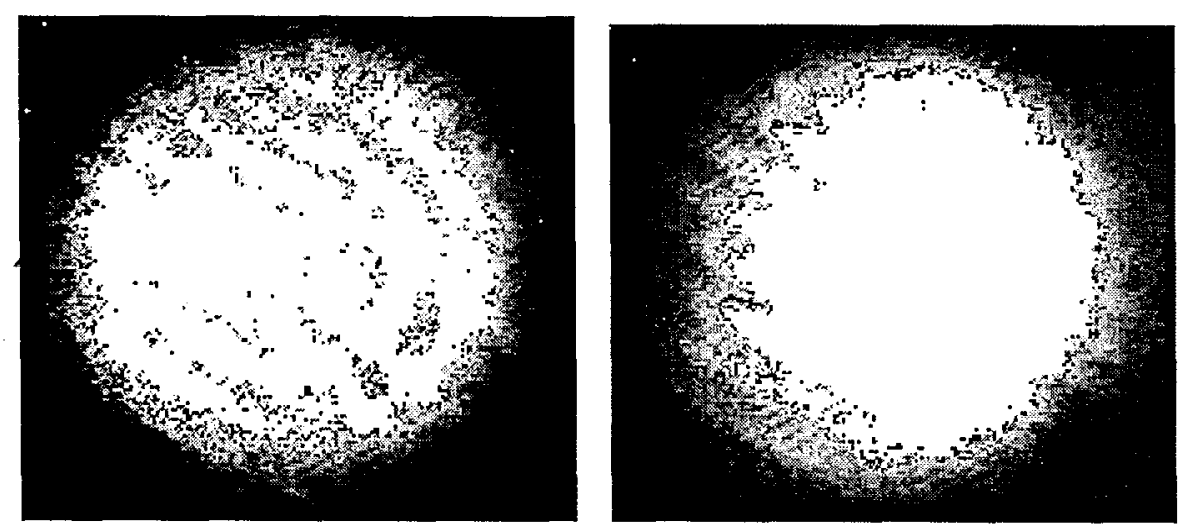

Figure 2. Near field beam profiles, left: preamp level power; right: amplified beam, 18 Watts 

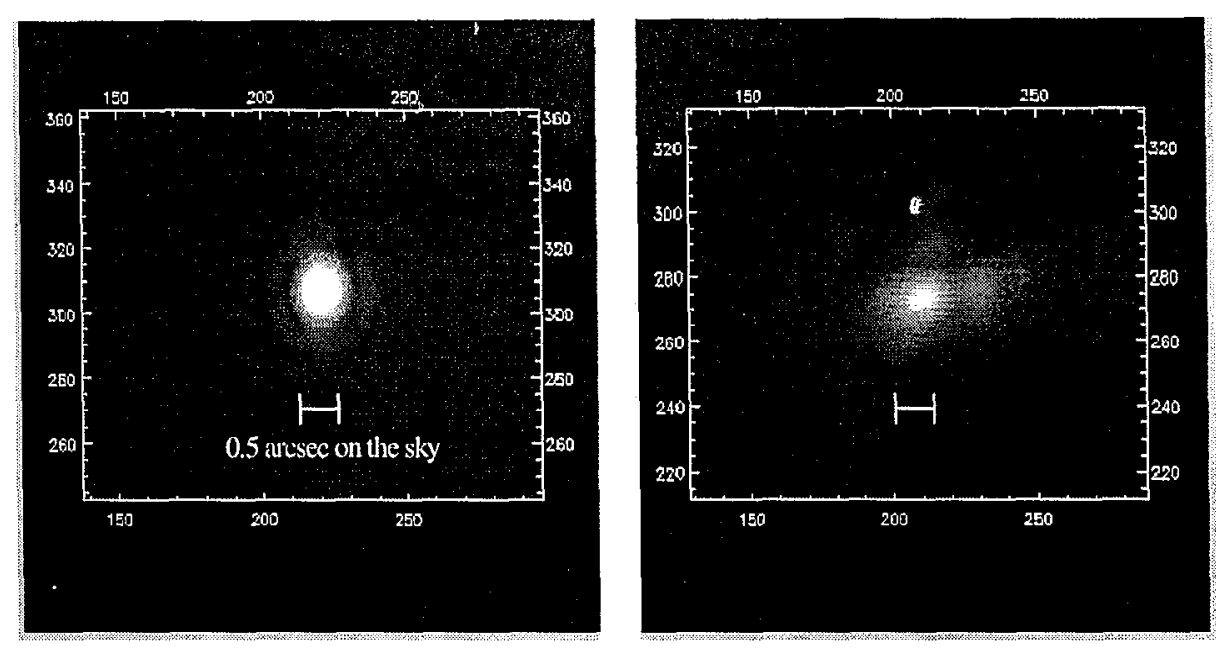

Figure 3. Far field beam profiles, left: preamp level power, right: amplified beam, 18 Watts the mesospheric sodium layer. As the laser projection telescope is taken through focus, astigmatism becomes apparent.

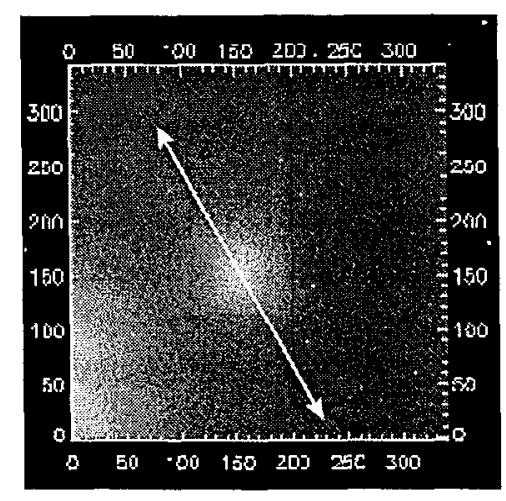

Inside focus

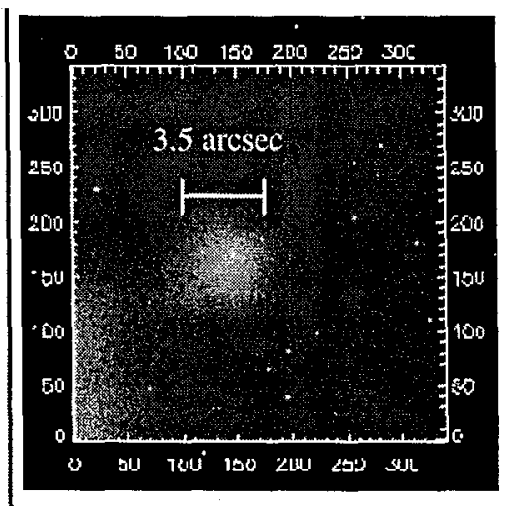

Circle of least confusion

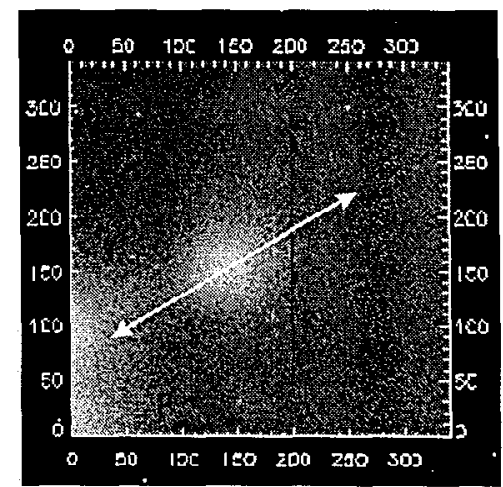

Outside focus

Figure 4. Guide star on the sky, as imaged through the receiving telescope focused on the mesospheric sodium layer. As the laser projection telescope is taken through focus, astigmatism becomes apparent. 


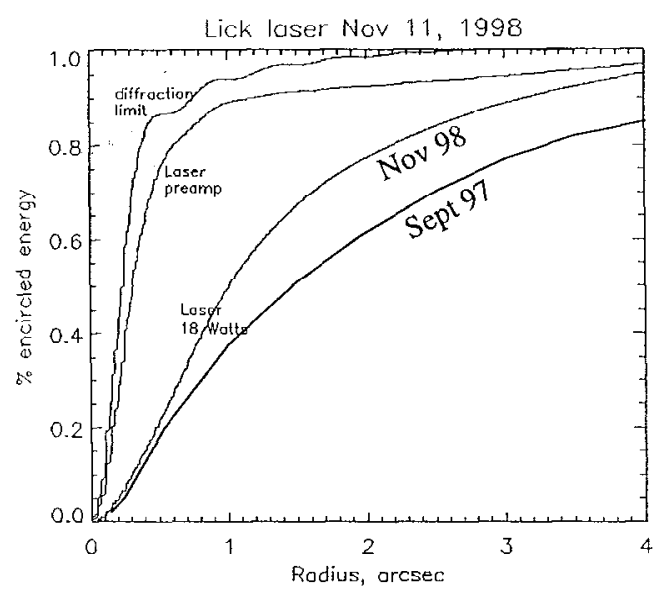

Figure 5. Encircled energy curves for the laser guide star.

\section{CONCLUSION}

There have been significant improvements made to the Lick guide star laser this past year, which resulted in a guide star spot about $30 \%$ smaller. Spot size reduction accounts in part for the record high laser guide star $\mathrm{AO}$ system Strehl (0.42 at 2.2 microns) in the November run.

There is still a clear discrepancy however between the expected spot size given the measured laser beam profile and the spot size as seen on the sky. The astigmatism apparent in the image of the spot in the sodium layer, and not apparent on the outgoing beam, is attributable to misalignment of the laser launch telescope, L2, L3. A subsequent ray trace analysis of the launch telescope revealed significant sensitivity to alignment through the center of these optics. Earlier pointing moves to place the laser guide star spot at the centerline of the AO system may have caused the misalignment.

The problem can be rectified by properly aligning the beam through center of the L2 and L3 lenses while at the same time fointing the laser to the desired direction on the sky. An iterative pointing/alignment technique using the existing hardware has been worked out and will be tested during engineering runs this year. It may also be possible to add an additional motorized degree of freedom in the laser beam path and use a control algorithm to decouple beam alignment from pointing/centering moves. This later remedy is presently under investigation.

\section{ACKNOWLEDGMENT}

This work was performed under the auspices of the U. S. Department of Energy by the Lawrence Livermore National Laboratory under contract number W-7405-ENG-48. It was supported by Laboratory Directed Rcsearch and Development funding within the University Relations Program and the Lasers Directorate. This support is gratefully acknowledged 


\section{REFERENCES}

1. Olivier, S. S., D. T. Gavel, H. W. Friedman, C. E. Max, J. R. An, K. Avicola, B. J. Bauman, J. M. Brase, E. W. Campbell, C. J. Carrano, J. B. Cooke, G. J. Freeze, E. L., Gates, V. K. Kanz, T. C. Kuklo, B. A. Macintosh, M. J. Newman, E. L. Pierce, K. E. Waltjen, J. A. Watson, Improved performance of the laser guide star adaptive optics system at Lick Observatory, Proc. SPIE 3762 , July 1999 [this conference].

2. Gavel, D. T. and H. W. Friedman, Measurements of the Lick Observatory sodium laser guide star, Proc. SPIE 3350, July 1998, 793-799.

3. Friedman, H. W., Erbert, G. V., Kuklo, T. C., Salmon, J. T., Smauley, D. A., Thompson, G. R., Malik, J. G., Wong, N. J., Kanz, V. K., Neeb, K., Sodium beacon laser system for the Lick Observatory, Proc. SPIE Vol. 2534, August 1995, 150-160.

4 Friedman, H. W., Cooke, J. B., Danforth, P. M., Erbert, G. V., Feldman, M., Gavel, D. T., Jenkins, S. L., Jones, H. E., Kanz, V. K., Kuklo, T. C., Newman, M. J., Pierce, E. L., Presta, R. W., Salmon, J. T., Thompson, G. R., Wong, N. J., Design and performance of a laser guide star system for the Keck II telescope, Proc. SPIE Vol. 3353, September, 1998, 260-276. 